\title{
25 Research Soure \\ Metformin Protects Lens Epithelial Cells Against Senescence in Naturally Aged Model of Mouse
}

\author{
Mengmeng Chen \\ Harbin Medical University \\ Yushan Fu \\ Harbin Medical University \\ Xu Wang \\ Xixi Hospitai of Hangzhou

\section{Ruitong Wu} \\ Harbin Medical University \\ Dongmei Su
}

National Research Institute for Family Planning

Nan Zhou

Harbin Medical University

Yanhua Qi ( qyh86605643@126.com )

Harbin Medical University https://orcid.org/0000-0001-5808-6837

\section{Research article}

Keywords: Metformin, Age-related cataract, AMPK, Autophagic flux, Senescence

Posted Date: April 19th, 2021

DOI: https://doi.org/10.21203/rs.3.rs-414664/v1

License: (c) (i) This work is licensed under a Creative Commons Attribution 4.0 International License. Read Full License

Version of Record: A version of this preprint was published at Cell Death Discovery on January 10th, 2022. See the published version at https://doi.org/10.1038/s41420-021-00800-w. 


\section{Abstract}

Background: The senescence of lens epithelial cells (LECs) is a major factor of age-related cataract (ARC). ARC results in visual impairment and severe vision loss in elderly. However, the specific mechanism of ARC is not yet clear and there are no effective therapeutic agents to halt the formation of ARC. The current study aimed to further explore the underlying mechanism of formation of ARC and investigate the potential anti-aging effect of metformin (MET) on ARC.

Methods: Male C57BL/ 6 mice were divided into three groups: the young control group (Young, 3 months, $n=40$ ), the naturally aged group (Aged, 20months, $n=60$ ) and MET group (MET, 20 months, $n=60)$. The young control group (Young, 3 months, $n=40$ ) and the naturally aged group (Aged, 20months, $n=60$ ) mice received ad libitum standard purified mouse diet and water, while the MET group mice were fed on chows supplied with $0.1 \%$ MET for 10 months. The transparency of lens and age-associated proteins P21, P53 were assessed in LECs in three groups. Furthermore, we determined the expressions of AMPK pathway and the effect of MET on AMPK pathway in LECs during the aging process of ARC. Besides, the relationship of autophagy and the senescence of in LECs and the role of MET in the autophagy in in LECs during the aging process of ARC were examined.

Results: Our results indicated that age-related inactivation of AMPK pathway and age-related impairment of autophagy might contribute to the senescence of in LECs and occurrence of ARC. Importantly, these results demonstrated that MET effectively alleviated the senescence of in LECs and the formation of ARC probably via inactivation of AMPK pathway and augmentation of autophagy.

Conclusion: These findings reveal that MET can be exploited as a potentially useful drug for ARC prevention. Our study will be informative for development of innovative strategies to the clinical treatment of ARC.

\section{Introduction}

Lens epithelial cells (LECs) are monolayer of cells lining the anterior capsule and extend to the equatorial lens bow ${ }^{[1,2]}$. The normal construction and function of LECs is essential for the maintenance of the transparency and metabolic homeostasis of the entire lens ${ }^{[3]}$. Accumulating evidence indicate that the senescence of LECs could cause modification, denaturation, aggregation of lens proteins including enzyme and crystallins, thereby ultimately leading to lens opacification or even cataract ${ }^{[3-5]}$. Cataract, especially age-related cataract (ARC), remains the leading cause of visional disability and blindness, which severely affects the quality of life of elderly individuals ${ }^{[6]}$. Unfortunately, up to date, there are no effective strategies in preventing ARC progression. Therefore, it is urgent to develop a pharmacological intervention that can serve transparency lens well and delay ARC progression.

Over the past few decades, scientists have achieved remarkable progress in improving health and extending lifespan using several genetic, dietary and pharmacological interventions ${ }^{[7]}$. One of the 
extensively studied anti-aging agents is metformin (MET). Substantial studies suggest that MET can alleviate senescence and extend healthy lifespan in various animal model systems. MET can not only prolong the lifespan of $C$. elegans, but also enhance the health and vitality of $C$. elegans ${ }^{[8]}$. MET can reduce age-related and oxidative stress-related accumulation of DNA damage in drosophila midgut stem cell to extend the lifespan ${ }^{[9]}$. Previous studies also indicate that chronic low-dose MET administration improve health and lifespan in mice ${ }^{[10]}$. Moreover, emerging evidence indicates that diabetic individuals treated with MET manifest a survival benefit even when compared to non-diabetic controls ${ }^{[11]}$.

In addition to the lifespan-promoting activity of MET in various model organisms, MET targets multiple cellular signaling pathways to ameliorate age-related diseases. MET has been shown to modulate autophagy mainly via AMP-activated protein kinase (AMPK) activation, as well as prevent neurodegeneration and neuroinflammation to play a neuroprotective role in Parkinson's disease [12]. Likewise, MET ameliorates age-related changes in the liver sinusoidal endothelial cell via AMPK and endothelial nitric oxide pathways ${ }^{[13]}$. Moreover, chronic AMPK activation by MET prevents cardiomyopathy by upregulating autophagy activity in diabetic OVE26 mice ${ }^{[14]}$. Besides, MET alleviates age-associated cardiovascular deterioration through enhancing autophagy and caloric restriction ${ }^{[15]}$. Furthermore, MET treatment can inhibit age-associated atherosclerosis in ApoE-/- mice and rabbit induced by high-fat diet, significantly preventing atherosclerotic plaque calcification, reducing highsensitivity C-reactive protein, and repressing the activation of NF-KB pathway in blood vessels ${ }^{[16-18]}$.

Based on these observations, the present study attempted to explore the possibility of that MET might also be able to delay the ARC aging process and the underlying molecular mechanism. We presented our findings that (i) chronic low-dose MET treatment could delay the senescence of LECs in naturally aged mice, thereby inhibiting lens opacity, (ii) AMPK pathway was inactivated and autophagic flux was impaired in senescent LECs in naturally aged mice, (iii) chronic low-dose MET treatment could delay the senescence of LECs in naturally aged mice by activating the AMPK pathway and enhancing autophagy. The present findings substantiate the putative anti-aging role of MET and provide sufficient evidence that MET may stand as a possible therapeutic option for ARC.

\section{Materials And Methods}

\subsection{Reagents}

MET was purchased from Bristol-Myers Squibb Company (China), Antibodies against p21 (ab188224), p53 (ab131442), phospho-AMPKa (Thr172) (ab133448), AMPKa1 (ab32047), $\beta$-actin (ab8226) and SQSTM1/p62 (3340-1) were obtained from Abcam (MA, USA). Antibodies against LC3 (12741), phosphoACC (Ser79) (3661) were purchased from Cell Signalling Technology (MA, USA).

\subsection{Animals and treatment}


Male C57BL6J mice of 3-month-old and Male C57BL6J mice of 8-month-old used in this study were purchased from Beijing Vital River Laboratory Animal Technology Co. Ltd. (Beijing, China). The mice were housed in temperature controlled (temperature $2025^{\circ} \mathrm{C}$ and relative humidity $55 \pm 15 \%$ ) conditions with a $12 \mathrm{~h} \mathrm{light/dark} \mathrm{cycle,} \mathrm{with} \mathrm{free} \mathrm{access} \mathrm{to} \mathrm{diet} \mathrm{and} \mathrm{water.} \mathrm{All} \mathrm{procedures} \mathrm{and} \mathrm{protocols} \mathrm{conducted} \mathrm{on}$ animals were approved by the ethics committee of Harbin Medical University.

The mice were divided into following three groups:

Young control group: Mice (Young, 3 months, $n=40$ ) received ad libitum standard purified mouse diet and water.

Naturally aged group: Mice (Aged, 20 months, $n=60$ ) received ad libitum standard purified mouse diet and water.

MET group: Mice (MET, 20 months, $n=60$ ) were maintained on a standard purified mouse diet and water until they reached 10 months of age when the treatments started. Next, those in the MET group were fed with the diet supplemented with $0.1 \%$ MET (Bristol Myers Squibb, China) and water freely for 10 months.

\subsection{Isolation of lenses and lens capsules}

The mice were euthanized by cervical dislocation. The whole eye was excised immediately from all the experimental animals, and placed in sterile saline. The optic nerve was placed upwards and fixed with tweezers. An incision is then made on the optic nerve that enters the eye, and the sclera is pulled back to expose the lens. Next, we used tweezers to gently remove the ciliary body fragments attached to the equatorial plane of the lens. Fresh lens were immediately taken photos and quickly fixed with $4 \%$ paraformaldehyde at room temperature. The entire lens capsules were quickly peeled from the remaining lens, snap-frozen in liquid nitrogen and stored at $-80^{\circ} \mathrm{C}$ for subsequent experiments.

\subsection{Haematoxylin and eosin (H\&E)}

Fresh lens were immediately fixed with $4 \%$ paraformaldehyde at room temperature and embedded in paraffin. For histological examination, the tissues were cut into $4 \mu \mathrm{m}$ slices. After deparaffinization and rehydration, the prepared paraffin sections were stained with hematoxylin solution for 8 min followed by 5 dips in $1 \%$ acid ethanol $(1 \% \mathrm{HCl}$ in $70 \%$ ethanol) and then rinsed in distilled water. Then the sections were stained with eosin solution for 3 min and followed by dehydration with graded alcohol and clearing in xylene. The morphological changes were observed under a microscope (Nikon, Eclipse, Japan).

\subsection{Immunohistochemistry (IHC) staining}

Five representative sections from each of the ten selected lens per group were used for immunohistochemistry (IHC) to assess expression of P21, P53, p-AMPK, p-ACC, LC3 and P62. After the prepared samples were incubated at $60^{\circ}$ for $2 \mathrm{~h}$, they were passed through xylene and graded alcohol series for deparaffinization. Next, the samples were boiled in $0.1 \mathrm{M}$ citric acid (pH 6.1) for $30 \mathrm{~min}$ and cooled to room temperature. After washing three times with PBS, the samples were soaked with $0.3 \%$ 
$\mathrm{H}_{2} \mathrm{O}_{2}$ to inhibit endogenous peroxidase activity. Subsequently, primary antibodies were added and cooled at $4^{\circ} \mathrm{C}$ overnight. The corresponding secondary antibodies were incubated for $1 \mathrm{~h}$ at $37^{\circ} \mathrm{C}$. After washing with PBS, the samples were incubated in diaminobenzidine (DAB) until the desired stain intensity developed. Images of the immunostained cells were captured using the Nikon Eclipse microscope (Nikon, Eclipse, Japan).

\subsection{Western blot analysis}

Total protein from mice were extracted in RIPA lysed buffer with protease inhibitor cocktail (Pierce, USA) and a BCA kit (Thermo Scientific, USA) was used to quantify the protein concentration. The total protein $(40 \mu \mathrm{g})$ was loaded for sodium dodecyl sulfate-polyacrylamide electrophoresis (SDS-PAGE) gel and electrophoretically transferred onto polyvinylidene difluoride filter (PVDF) membranes (Millipore, Germany). Membranes were incubated overnight with primary antibody at $4^{\circ} \mathrm{C}$ overnight. Next day, the membranes were incubated with corresponding secondary antibodies for $1 \mathrm{~h}$ at $37^{\circ} \mathrm{C}$. The immunoreactive bands were visualized using the Chemiluminescent Substrate method with a Super Signal West Pico kit (Pierce Biotechnology, USA). Three independent experiments were performed.

\subsection{Statistical analysis.}

The data were expressed as the means \pm standard deviation (SD). Statistical analyses were conducted using GraphPad Prism 7.0 software and Microsoft Excel. Protein quantitative was analyzed using Image $\mathrm{J}$ software. Student t-test and analysis of variance (ANOVA) were used to calculate the statistical significance of the experimental data. Differences with a P-value $<0.05$ were considered to indicate statistically significant differences. All experiments were repeated independently at least 3 times.

\section{Results}

\subsection{Senescence of lens epithelial cells was associated with the ARC in naturally aged mice}

Multiple potential mechanisms of ARC, including the effect of aging, have been extensively investigated. However, the association between senescence of LECs and ARC in vivo is far from completely

understood. We surmised that senescence of LECs may result in ARC in vivo. Therefore, we constructed a mouse model of ARC. Our results showed that the transparency of lens in naturally aged mice (Aged) was significantly decreased as compared with that in the Young control group (Young) (Fig. 1a). HE staining assay indicated that the LECs in the Young control group (Young) were round, arranged regularly and the density of the normal LECs was relative high, while the LECs in the naturally aged mice (Aged) were flat, arranged unevenly and the density of senescent LECs decreased markedly (Fig. 1b). Then, we tested the protein expression of senescence-associated markers via IHC staining and Western Blot analysis. IHC staining revealed that the expressions of P21 and P53 were increased in LECs in the naturally aged mice (Aged) than that in LECs in the Young control group (Young) (Fig. 1c, d). Similarly, we further found that the protein expressions of P21 and P53 were obviously higher in the lens capsules in the naturally aged 
mice (Aged) than that in the Young control group (Young) (Fig. 1e). These results suggested that senescence of LECs was associated with the ARC in naturally aged mice.

\subsection{Chronic low-dose MET administration served the transparency of lens and alleviated the senescence of lens epithelial cells.}

Subsequently, chronic low-dose MET administration was performed to explore whether MET could inhibit lens opacity and relieve age-related senescence of LECs in naturally aged mice. As expected, the transparency of lens in the MET group (MET) was greatly improved compared with the transparency of lens in the naturally aged mice (Aged) (Fig. 2a). HE staining assay demonstrated that the density of LECs in the MET group (MET) was notably increased compared with that in the naturally aged mice (Aged) (Fig. 2b). Moreover, the LECs in the MET group (MET) were relatively round compared with that in the naturally aged mice (Aged) (Fig. 2b). IHC examination showed that the expressions of P21 and P53 were decreased in LECs in the MET group (MET) than that in LECs in the naturally aged mice (Aged) (Fig. 2c, d). Analogously, Western Blot analysis showed that the protein expressions of P21 and P53 were remarkably decreased in the lens capsules in the MET group (MET) compared with that in the lens capsules in the naturally aged mice (Aged) (Fig. 2e). These results signified that chronic low-dose MET administration could serve the transparency of lens and alleviate the senescence of lens epithelial cells.

\subsection{The AMPK pathway was inactivated in lens epithelial cells of naturally aged mice}

An overwhelming amount of data supports the conclusion that activating AMPK is sufficient to extend lifespan in model organisms, increasing its appeal as a longevity target ${ }^{[19,20]}$. Taken this theory into consideration, we assessed the expression of AMPK pathway in LECs of mice. As shown in Fig. 3a and $3 b$, the expressions of phosphorylated AMPKa (Thr172) and phosphorylated ACC (Acetyl CoA carboxylase) (Ser79) were evidently reduced in LECs of in the naturally aged mice (Aged) compared with that of the Young control group (Young). In addition, Western Blot analysis showed that the protein expressions of phosphorylated AMPKa (Thr172) and phosphorylated ACC (Ser79) were significantly down-regulated in the lens capsules in the naturally aged mice (Aged), while the total protein levels of AMPKa and ACC remained unchanged (Fig. 3c). These data revealed that the AMPK pathway was inactivated in LECs of naturally aged mice.

\subsection{Chronic low-dose MET administration attenuated the senescence of lens epithelial cells in naturally aged mice via AMPK activation}

To better clarify the mechanisms of MET in alleviating the senescence of LECs, we examined the effect of MET on AMPK pathway in LECs of mice. IHC staining indicated that the expressions of phosphorylated AMPKa (Thr172) and phosphorylated ACC (Ser79) were elevated in LECs in the MET group (MET) (Fig. 4a, b). Unsurprisingly, the protein levels of phosphorylated AMPKa (Thr172) and phosphorylated ACC (Ser79) were obviously raised in the lens capsules in the MET group (MET) (Fig. 4c). These results 


\subsection{Autophagic flux was impaired in lens epithelial cells of naturally aged mice}

Previous evidence raises the exciting possibility that autophagy may play an important role in combating the adverse effects of aging ${ }^{21,22]}$. Then, we determined the expressions of LC3and P62, as they were widely accepted as biomarkers of the levels of autophagy. Our results illustrated that there was a significant difference in the LC3-II/I ratio and expression of P62 between the naturally aged group and the Young control group (Fig. 5a, b). Consistent with this result, the protein levels of LC3-II and P62 were markedly increased in the lens capsules in the naturally aged group (Fig. $5 \mathrm{c}$ ). The above results strongly suggested that autophagic flux was impaired in LECs of naturally aged mice.

\subsection{Chronic low-dose MET administration restored the autophagic flux to attenuate the senescence of lens epithelial cells in naturally aged mice}

To further analyze the molecular and cellular changes in the lens after treatment with MET, we tested the expressions of the critical genes of autophagy in LECs of mice. IHC staining showed the levels of LC3-II/I and P62 were remarkably lowered in LECs in the MET group (Fig. 6a, b). At the same time, Western Blot analysis also showed that the protein expressions of LC3-II and P62 were significantly decreased in the lens capsules in the MET group (Fig. 6c). Taken together, we speculated that chronic low-dose MET administration might restore the autophagic flux to attenuate the senescence of LECs in naturally aged mice.

\section{Discussion}

Untreated cataract is the leading cause of blindness worldwide, which is characterized by opacification of the crystalline lens ${ }^{[23]}$. Epidemiological studies have shown that age is the dominant risk for cataracts, known as ARC, and the only treatment is surgical removal [24]. Previous studies have shown that senescence of LECs is a main cause of ARC [25]. Until now, there are no effective therapeutic agents to inhibit the cloudiness of lens and attenuate the senescence of LECs without undesirable side effects. Exploring the molecular biological basis of the senescence of lens epithelial cells, and the mechanism of protecting normal LECs against senescence are the focus of present study. Our results showed that inactivation of AMPK and impairment of autophagy were associated with senescence of LECs and ARC. Furthermore, we found that MET might protect LECs against senescence and inhibit the cloudiness of lens via activation of AMPK and improvement of autophagy.

Senescence is a fundamental biological process accompanied by a general decline in tissue function. Indeed, as the lifespan increases, age-related dysfunction, such as ARC, has become a growing public health issue ${ }^{[26]}$. Senescence of LECs has been documented involved in the occurrence and the 
development of ARC ${ }^{[5]}$. Our results showed that the LECs in the naturally aged mice (Aged) were flat, arranged unevenly and the density of LECs decreased markedly (Fig. 1b). Additionally, the expressions of aging-associated gene (P21, P53) were evidently up-regulated in the LECs in the naturally aged mice (Aged) (Fig. 1c, d, e). These results above implied that the senescence of LECs was associated with the occurrence and the development of ARC. Thus, an accurate understanding of the molecular mechanisms of LECs senescence may be required to provide a rationale for new treatment strategies.

MET, a biguanide, is the most widely-prescribed oral hypoglycemic medication for type 2 diabetes worldwide. Emerging evidence indicates that MET has beneficial effects on health beyond those associated with amelioration in glycemia ${ }^{[27]}$. It has been confirmed that the mechanisms of MET in targeting fundamental pathways in biological aging, including activation of AMPK, augmenting autophagy, inhibiting inflammation, exerting antioxidative effects ${ }^{[28]}$. In humans, MET has been in clinical use for over 60 years, has a high safety profile and is uniquely positioned to intervene several crucial pathways responsible for aging and age-related diseases. However, the effects of MET on ARC and the regulatory mechanism of those effects have never been reported. Consequently, chronic low-dose MET administration was innovatively performed on ARC in naturally aged mice. Our results demonstrated that chronic low-dose MET administration was able to serve transparency lens (Fig. 2a) and attenuate the senescence of LECs in vivo (Fig. 2b, c).

It is widely acknowledged that MET activates AMPK pathway, which is the potential mechanism of MET to exert anti-aging effect ${ }^{[29]}$. AMPK, cellular energy sensor, plays a crucial role in regulating cellular energy balance ${ }^{[30]}$. Several studies have illustrated that AMPK serves as an integrator and mediator of several pathways and processes linking energetics to longevity. In our study, we found that the inactivation of AMPK was the feature of senescent LECs in naturally aged mice (Fig. 3). Later, MET was shown to stimulate AMPK pathway to prevent the senescence of LECs (Fig. 4). As an established AMPK activator, MET's role in aging prevention is generally attributed to its effects on modulating downstream signaling pathways, such as restoration of autophagy, activation of Sirt1 and Foxo1, as well as the suppression of $\mathrm{mTOR}^{[31,32]}$. Our study provided a new line of evidence stressing the importance of AMPK function activated by MET on preventing the occurrence of ARC.

As mentioned above, AMPK thus communicates with numerous pathways and proteins to exert the antisenescence effect, including augmentation of autophagy. Autophagy, emerging as a core process for longevity assurance, has attracted widespread interest as a potential therapeutic target for ageassociated diseases ${ }^{[21]}$. Autophagy is a fluid, multi-step complex biological process. The complete process of autophagy is called autophagic flux, which is widely used to reflect the level of autophagy. As an indicator of autophagy, LC3-II is tightly bound to autophagosomal membrane, which is degraded by lysosomal enzymes. Likewise, P62, autophagy-specific substrate, usually is degraded in autophagolysosome and its expression indirectly reflects the level of autophagy. In present study, Our findings implied that the level of autophagy was distinctly declined in senescent LECs (Fig. 5), while MET reversed of age-related impairment of autophagy (Fig. 6). With regard to the anti-aging molecular 
mechanism of MET stems from the primary function of AMPK in cells because it generally enhances autophagy via AMPK activation ${ }^{[33]}$. On the other hand, MET is shown to augment autophagy directly, thereby contributing to prevention of senescence ${ }^{[34]}$. However, more detailed studies are absolutely needed to elucidate the mechanism(s) that MET augments autophagy via independent or dependent AMPK pathway.

\section{Conclusion}

In summary, we provided evidence that inactivation of AMPK pathway and age-related impairment of autophagy might contribute to the senescence of LECs and occurrence of ARC. In addition, the data from our experiments demonstrated that MET effectively retarded the senescence of LECs and the formation of ARC via activation of AMPK pathway and augmentation of autophagy. Our findings is a basic line for further investigation of the molecular mechanisms in the role of MET during aging process of ARC, and will be informative for more intensive studies to the development of new strategies against formation and development of ARC.

\section{Abbreviations}

ARC, age-related cataract; MET, metformin; LECs, lens epithelial cells; H\&E, haematoxylin and eosin; IHC, immunohistochemistry; AMPK, adenosine monophosphate (AMP)-activated protein kinase; ACC, Acetyl CoA carboxylase; PVDF, Polyvinylidene fluoride; FBS, Fetal bovine serum.

\section{Declarations}

\section{Acknowledgements}

None.

\section{Author contributions}

Chen $\mathrm{M}$ designed experiments, carried out most of the experiments, analyzed the data, prepared the figures, and wrote the manuscript. Fu Y, Wang X, Wu R, Su D performed some experiments or contributed to data analysis and manuscript preparation. Zhou N, Qi Y contributed to study design.

\section{Funding}

This work was supported by the National Natural Science Foundation of China (No:81770912) awarded to Yanhua Qi.

\section{Data availability statements}

The datasets used and analysed during the current study are included in this published article. 
Consent for publication

Authors agreed to this publication.

Conflict of interest

The authors declare that they have no conflict of interest.

Ethics approval and consent to participate

This study was obeyed the Guide for the Care and Use of Laboratory Animals. All protocols were authorized by the Ethics Committee of Harbin Medical University.

Contributor Information

Mengmeng Chen, Email: 358434182@qq.com

Nan Zhou, Email: 10423588@qq.com.

Yanhua Qi, Email: qyh86605643@126.com

\section{References}

1. Lovicu FJ, McAvoy JW, de longh RU: Understanding the role of growth factors in embryonic development: insights from the lens. Philos Trans R Soc Lond B Biol Sci 2011, 366(1568):1204-1218.

2. Shiels A, Hejtmancik JF: Biology of Inherited Cataracts and Opportunities for Treatment. Annu Rev Vis Sci 2019, 5:123-149.

3. Bai J, Yang F, Dong L, Zheng Y: Ghrelin Protects Human Lens Epithelial Cells against Oxidative Stress-Induced Damage. Oxid Med Cell Longev 2017, 2017:1910450.

4. Rwei P, Alex Gong CS, Luo LJ, Lin MB, Lai JY, Liu HL: In vitro investigation of ultrasound-induced oxidative stress on human lens epithelial cells. Biochem Biophys Res Commun 2017, 482(4):954960.

5. Yan Y, Yu H, Sun L, Liu H, Wang C, Wei X, Song F, Li H, Ge H, Qian H et al: Laminin alpha4 overexpression in the anterior lens capsule may contribute to the senescence of human lens epithelial cells in age-related cataract. Aging (Albany NY) 2019, 11(9):2699-2723.

6. Asbell PA, Dualan I, Mindel J, Brocks D, Ahmad M, Epstein S: Age-related cataract. Lancet 2005, 365(9459):599-609.

7. Campisi J, Kapahi P, Lithgow GJ, Melov S, Newman JC, Verdin E: From discoveries in ageing research to therapeutics for healthy ageing. Nature 2019, 571(7764):183-192.

8. Onken B, Driscoll M: Metformin induces a dietary restriction-like state and the oxidative stress response to extend C. elegans Healthspan via AMPK, LKB1, and SKN-1. PLoS One 2010, 5(1):e8758. 
9. Na HJ, Park JS, Pyo JH, Lee SH, Jeon HJ, Kim YS, Yoo MA: Mechanism of metformin: inhibition of DNA damage and proliferative activity in Drosophila midgut stem cell. Mech Ageing Dev 2013, 134(9):381-390.

10. Martin-Montalvo A, Mercken EM, Mitchell SJ, Palacios HH, Mote PL, Scheibye-Knudsen M, Gomes AP, Ward TM, Minor RK, Blouin MJ et al: Metformin improves healthspan and lifespan in mice. Nat Commun 2013, 4:2192.

11. Holman RR, Paul SK, Bethel MA, Matthews DR, Neil HA: 10-year follow-up of intensive glucose control in type 2 diabetes. N Engl J Med 2008, 359(15):1577-1589.

12. Paudel YN, Angelopoulou E, Piperi C, Shaikh MF, Othman I: Emerging neuroprotective effect of metformin in Parkinson's disease: A molecular crosstalk. Pharmacol Res 2020, 152:104593.

13. Hunt NJ, Lockwood GP, Kang SWS, Pulpitel T, Clark X, Mao H, McCourt PAG, Cooney GJ, Wali JA, Le Couteur FH et al: The Effects of Metformin on Age-Related Changes in the Liver Sinusoidal Endothelial Cell. J Gerontol A Biol Sci Med Sci 2020, 75(2):278-285.

14. Xie Z, Lau K, Eby B, Lozano P, He C, Pennington B, Li H, Rathi S, Dong Y, Tian R et al: Improvement of cardiac functions by chronic metformin treatment is associated with enhanced cardiac autophagy in diabetic OVE26 mice. Diabetes 2011, 60(6):1770-1778.

15. Abdellatif M, Sedej S, Carmona-Gutierrez D, Madeo F, Kroemer G: Autophagy in Cardiovascular Aging. Circ Res 2018, 123(7):803-824.

16. Lamanna C, Monami M, Marchionni N, Mannucci E: Effect of metformin on cardiovascular events and mortality: a meta-analysis of randomized clinical trials. Diabetes Obes Metab 2011, 13(3):221228.

17. Hong J, Zhang Y, Lai S, Lv A, Su Q, Dong Y, Zhou Z, Tang W, Zhao J, Cui L et al: Effects of metformin versus glipizide on cardiovascular outcomes in patients with type 2 diabetes and coronary artery disease. Diabetes Care 2013, 36(5):1304-1311.

18. Cai Z, Ding Y, Zhang M, Lu Q, Wu S, Zhu H, Song P, Zou MH: Ablation of Adenosine MonophosphateActivated Protein Kinase alpha1 in Vascular Smooth Muscle Cells Promotes Diet-Induced Atherosclerotic Calcification In Vivo. Circ Res 2016, 119(3):422-433.

19. Burkewitz K, Zhang Y, Mair WB: AMPK at the nexus of energetics and aging. Cell Metab 2014, 20(1):10-25.

20. Jeon SM: Regulation and function of AMPK in physiology and diseases. Exp Mol Med 2016, 48(7):e245.

21. Wong SQ, Kumar AV, Mills J, Lapierre LR: Autophagy in aging and longevity. Hum Genet 2020, 139(3):277-290.

22. Shirakabe A, Ikeda Y, Sciarretta S, Zablocki DK, Sadoshima J: Aging and Autophagy in the Heart. Circ Res 2016, 118(10):1563-1576.

23. Lee CM, Afshari NA: The global state of cataract blindness. Curr Opin Ophthalmo/2017, 28(1):98103. 
24. Thompson J, Lakhani N: Cataracts. Prim Care 2015, 42(3):409-423.

25. Barzilai N, Crandall JP, Kritchevsky SB, Espeland MA: Metformin as a Tool to Target Aging. Cell Metab 2016, 23(6):1060-1065.

26. Yu M, Zhang H, Wang B, Zhang Y, Zheng X, Shao B, Zhuge Q, Jin K: Key Signaling Pathways in Aging and Potential Interventions for Healthy Aging. Cells 2021, 10(3).

27. Cai H, Han B, Hu Y, Zhao X, He Z, Chen X, Sun H, Yuan J, Li Y, Yang X et al: Metformin attenuates the Dgalactoseinduced aging process via the UPR through the AMPK/ERK1/2 signaling pathways. Int $J$ Mol Med 2020, 45(3):715-730.

28. Bharath LP, Agrawal M, McCambridge G, Nicholas DA, Hasturk H, Liu J, Jiang K, Liu R, Guo Z, Deeney $\mathrm{J}$ et al: Metformin Enhances Autophagy and Normalizes Mitochondrial Function to Alleviate AgingAssociated Inflammation. Cell Metab 2020, 32(1):44-55 e46.

29. Araujo AA, Pereira A, Medeiros C, Brito GAC, Leitao RFC, Araujo LS, Guedes PMM, Hiyari S, Pirih FQ, Araujo Junior RF: Effects of metformin on inflammation, oxidative stress, and bone loss in a rat model of periodontitis. PLoS One 2017, 12(8):e0183506.

30. Herzig S, Shaw RJ: AMPK: guardian of metabolism and mitochondrial homeostasis. Nat Rev Mol Cell Biol 2018, 19(2):121-135.

31. Ren $H$, Shao Y, Wu C, Ma X, Lv C, Wang Q: Metformin alleviates oxidative stress and enhances autophagy in diabetic kidney disease via AMPK/SIRT1-Fox01 pathway. Mol Cell Endocrinol 2020, 500:110628.

32. Yang F, Qin Y, Wang Y, Meng S, Xian H, Che H, Lv J, Li Y, Yu Y, Bai Y et al: Metformin Inhibits the NLRP3 Inflammasome via AMPK/mTOR-dependent Effects in Diabetic Cardiomyopathy. Int J Biol Sci 2019, 15(5):1010-1019.

33. Li Y, Chen Y: AMPK and Autophagy. Adv Exp Med Biol 2019, 1206:85-108.

34. Xu Y, Li Y, Ma L, Xin G, Wei Z, Zeng Z, Xing Z, Li S, Niu H, Huang W: d-galactose induces premature senescence of lens epithelial cells by disturbing autophagy flux and mitochondrial functions. Toxicol Lett 2018, 289:99-106.

\section{Figures}


a

$b$

Young

Aged
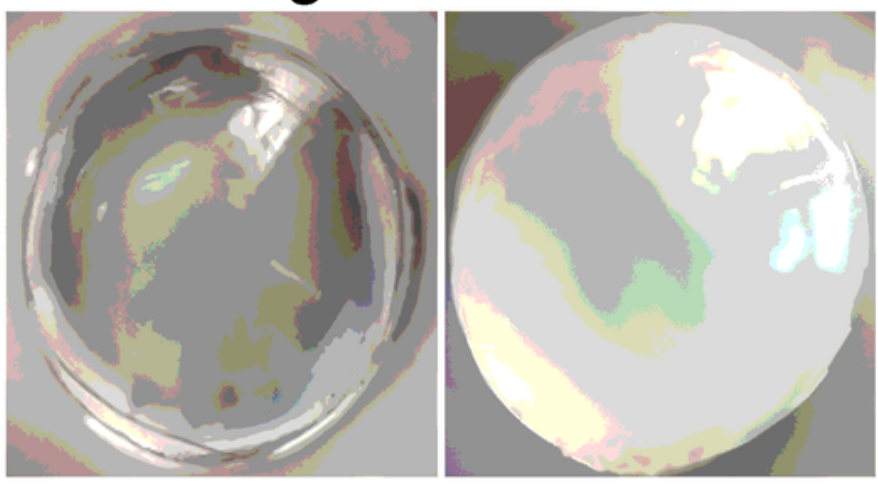

Young

Aged

C

d

\section{Young(P53) Aged(P53)}
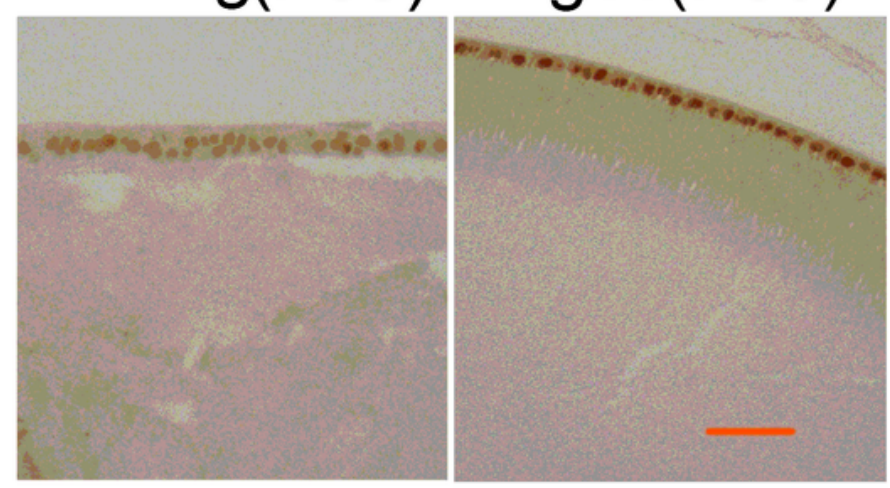

Young(P21) Aged(P21)

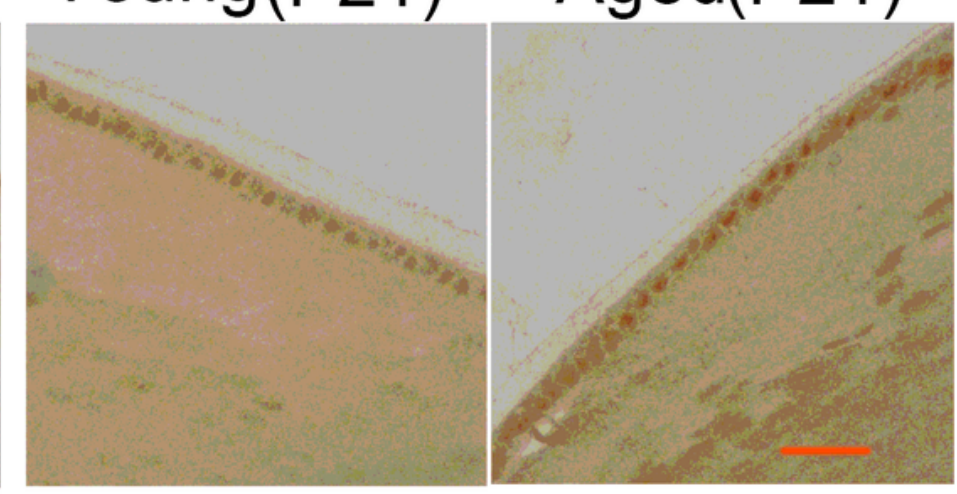

e
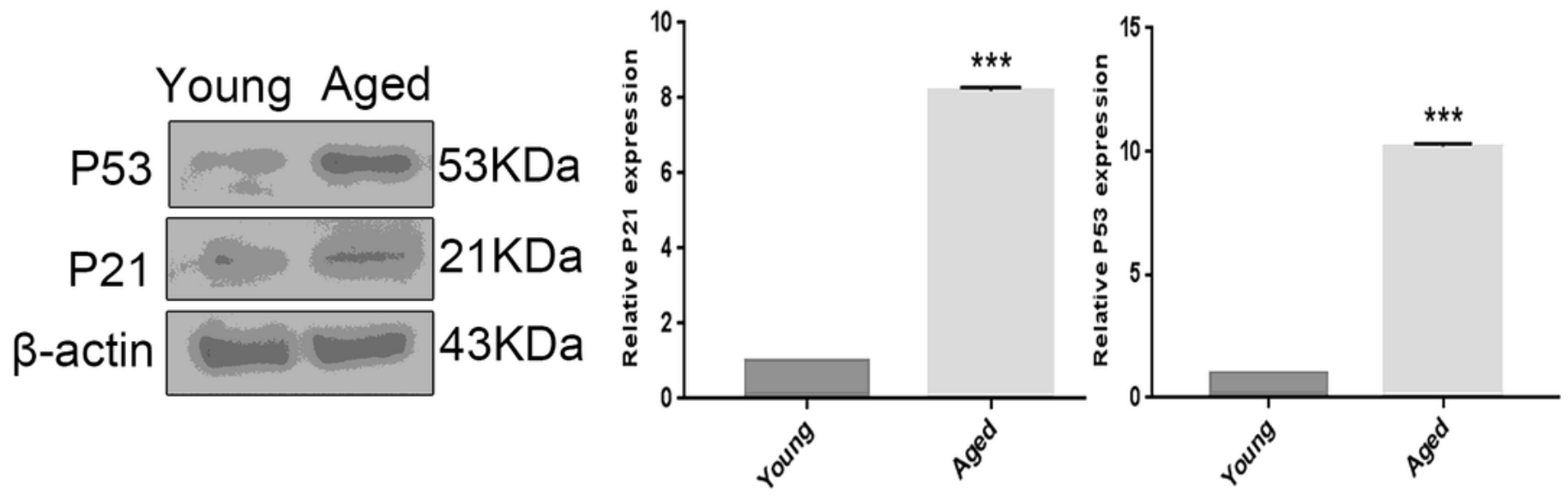

Figure 1

Senescence of lens epithelial cells was associated with the ARC in naturally aged mice. (a). Representative images of morphological observations of the lens in the Young (left) and the Aged (right). (b). The lens of the Young (left) and the Aged (right) were analysed by H\&E staining. (c). Representative images from IHC assays against P53 in the Young (left) and the Aged (right). (d). Representative images from IHC assays against P21 in the Young (left) and the Aged (right). (e). Western blot analysis of P53, 
P21 and $\beta$-actin in the Young and the Aged. Data were shown as mean \pm SD and are representative of 3 independent experiments. **, $p<0.01$; $* \star *, p<0.001$ compared to the Young. The bar represents $20 \rrbracket \mathrm{m}$.

a

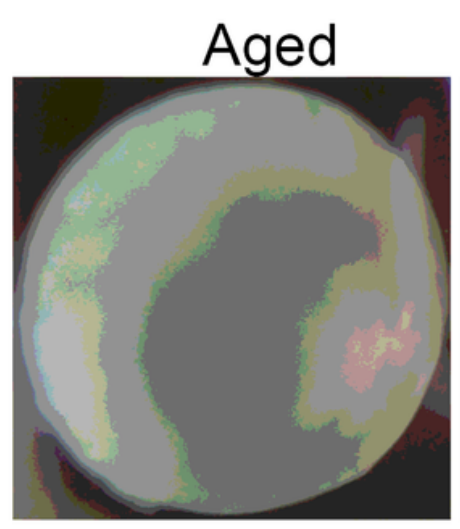

C

Aged(P53)

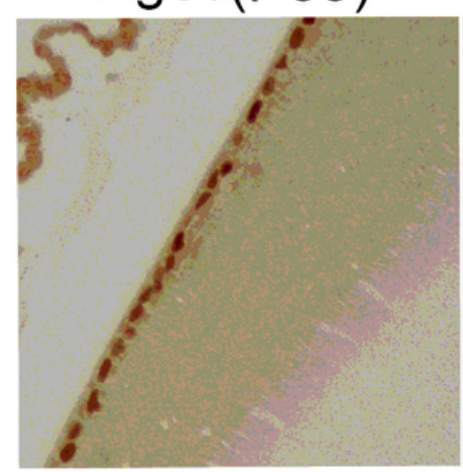

b

MET
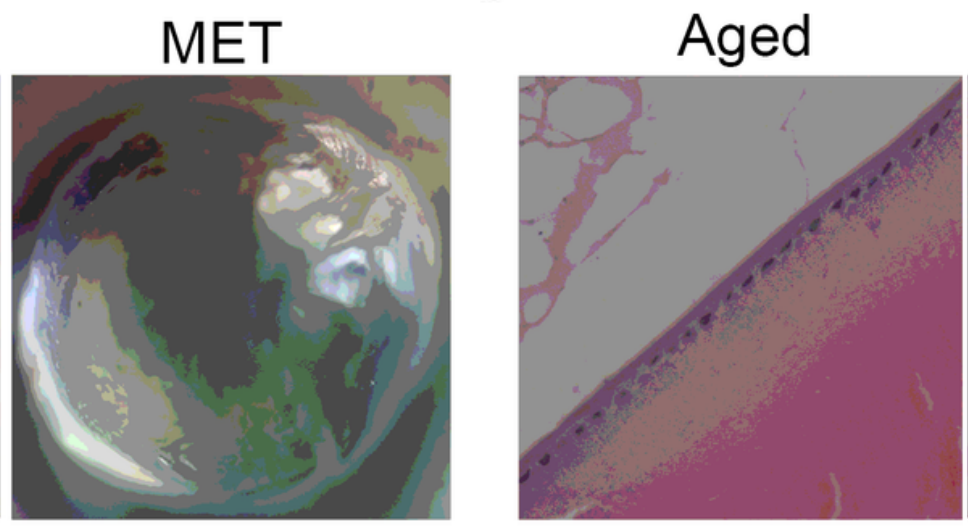

d
MET(P53)

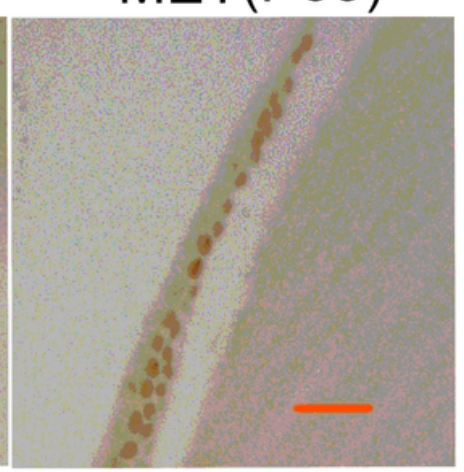

Aged(P21)

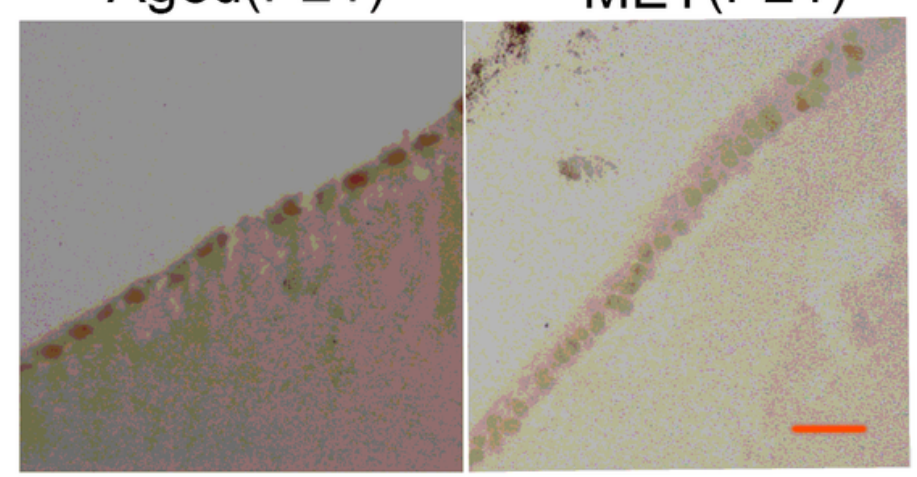

e
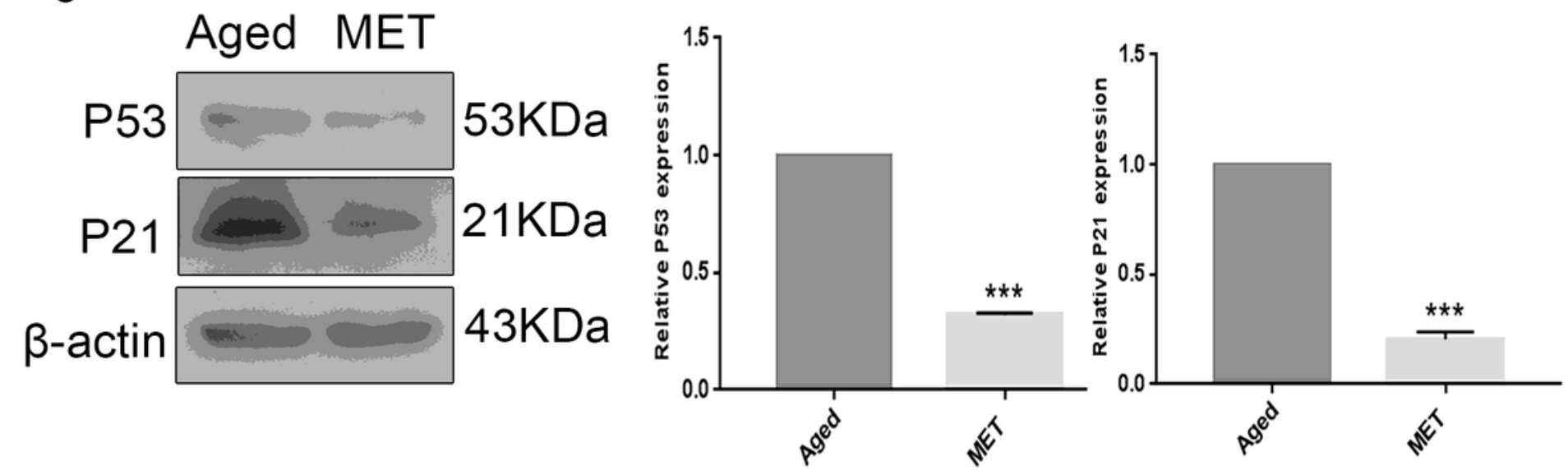

\section{Figure 2}

Chronic low-dose MET administration served the transparency of lens and alleviated the senescence of lens epithelial cells. (a). Representative images of morphological observations of lens in the Aged (left) and the MET (right). (b).The lens of the Aged (left) and the MET (right) were analysed by H\&E staining. (c). Representative images from IHC assays against P53 in the Aged (left) and the MET (right). (d). Representative images from IHC assays against P21 in the Aged (left) and the MET (right). (e). Western 
blot analysis of P53, P21 and $\beta$-actin in the Aged (left) and the MET (right). Data were shown as mean \pm SD and are representative of 3 independent experiments. ${ }^{* \star *}, p<0.001$ compared to the Aged. The bar represents $20 \bigotimes \mathrm{m}$.
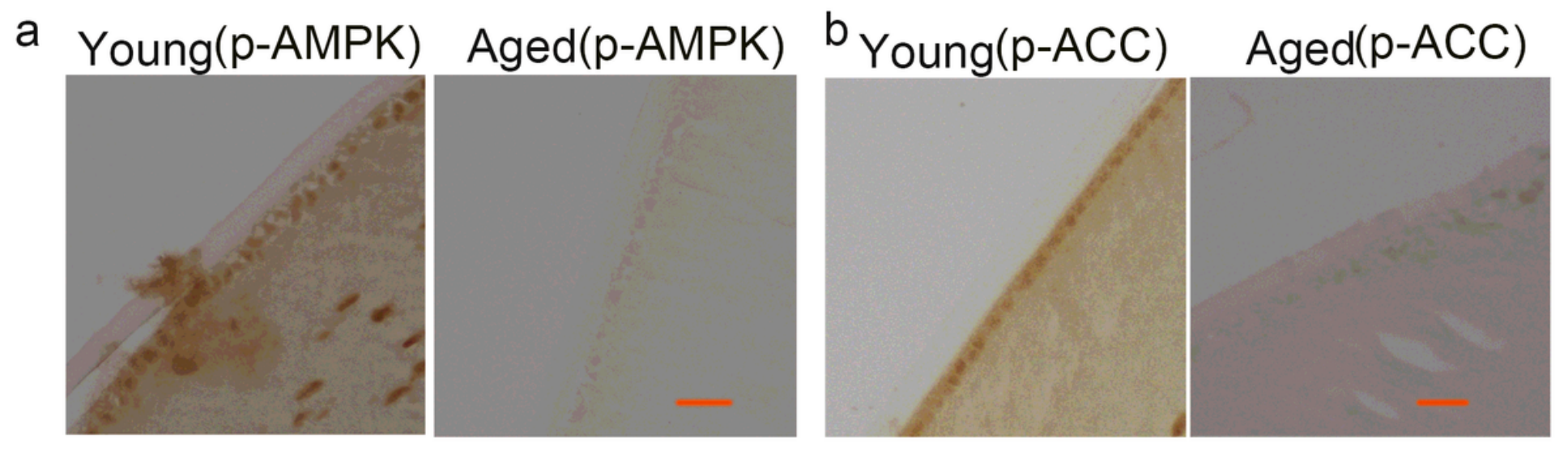

C
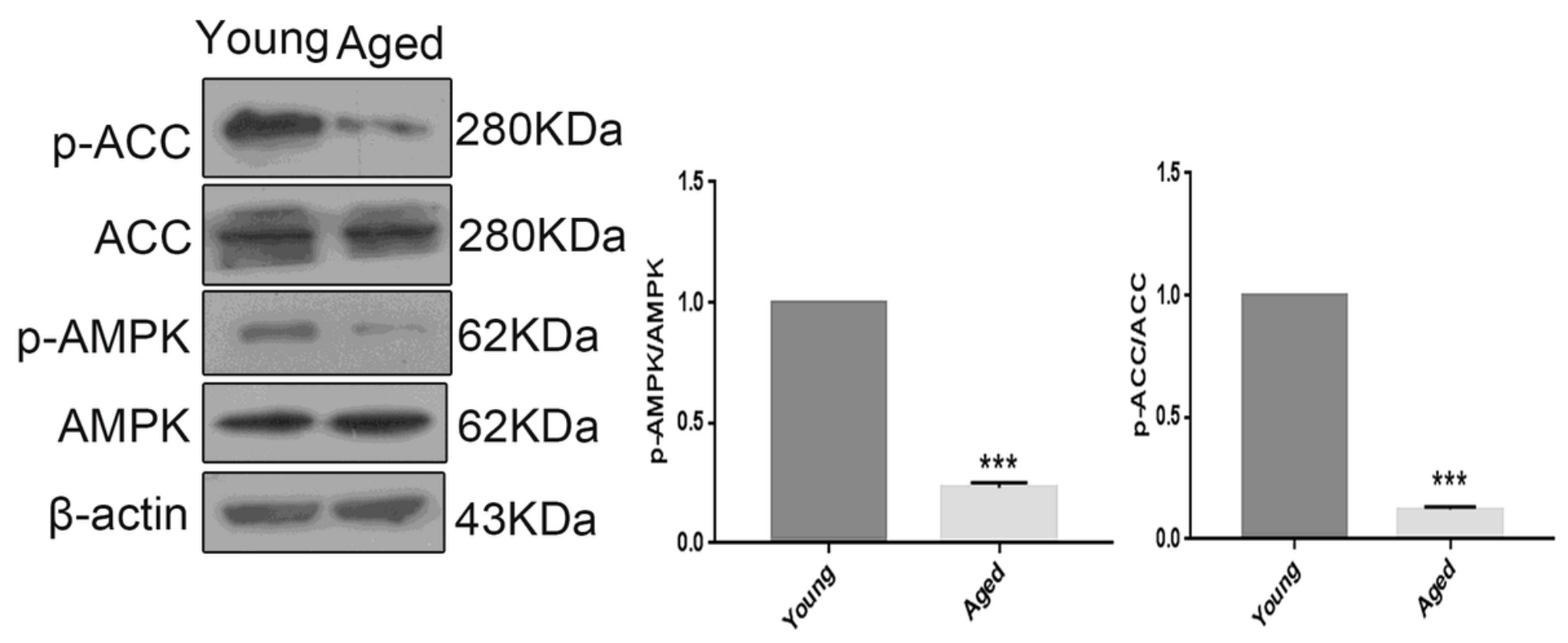

Figure 3

The AMPK pathway was inactivated in lens epithelial cells of naturally aged mice. (a). Representative images from IHC assays against p-AMPK in the Young (left) and the Aged (right). (b). Representative images from IHC assays against p-ACC in the Young (left) and the Aged (right). (c). Western blot analysis of AMPK, p-AMPK, ACC, p-ACC and $\beta$-actin in the Young and the Aged. Data were shown as mean \pm SD and are representative of 3 independent experiments. ${ }^{\star \star \star}, p<0.001$ compared to the Young. The bar represents $20 \bigotimes \mathrm{m}$. 


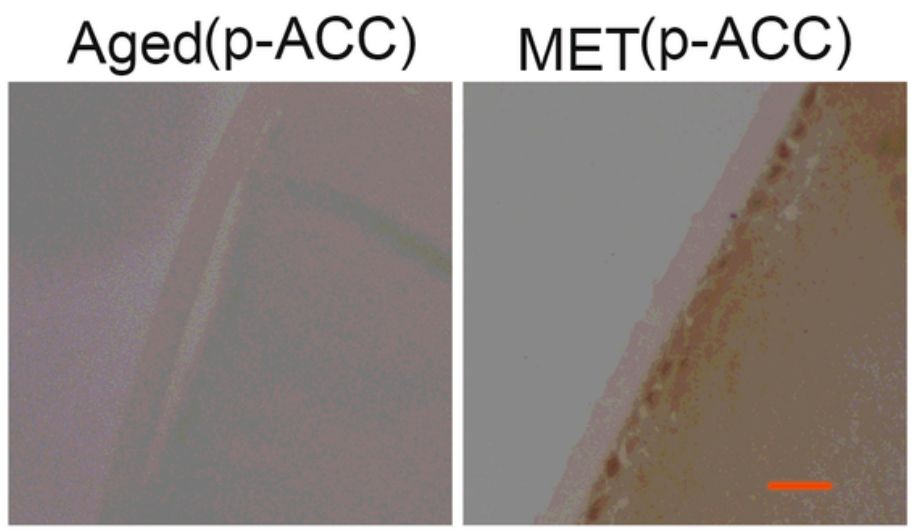

C
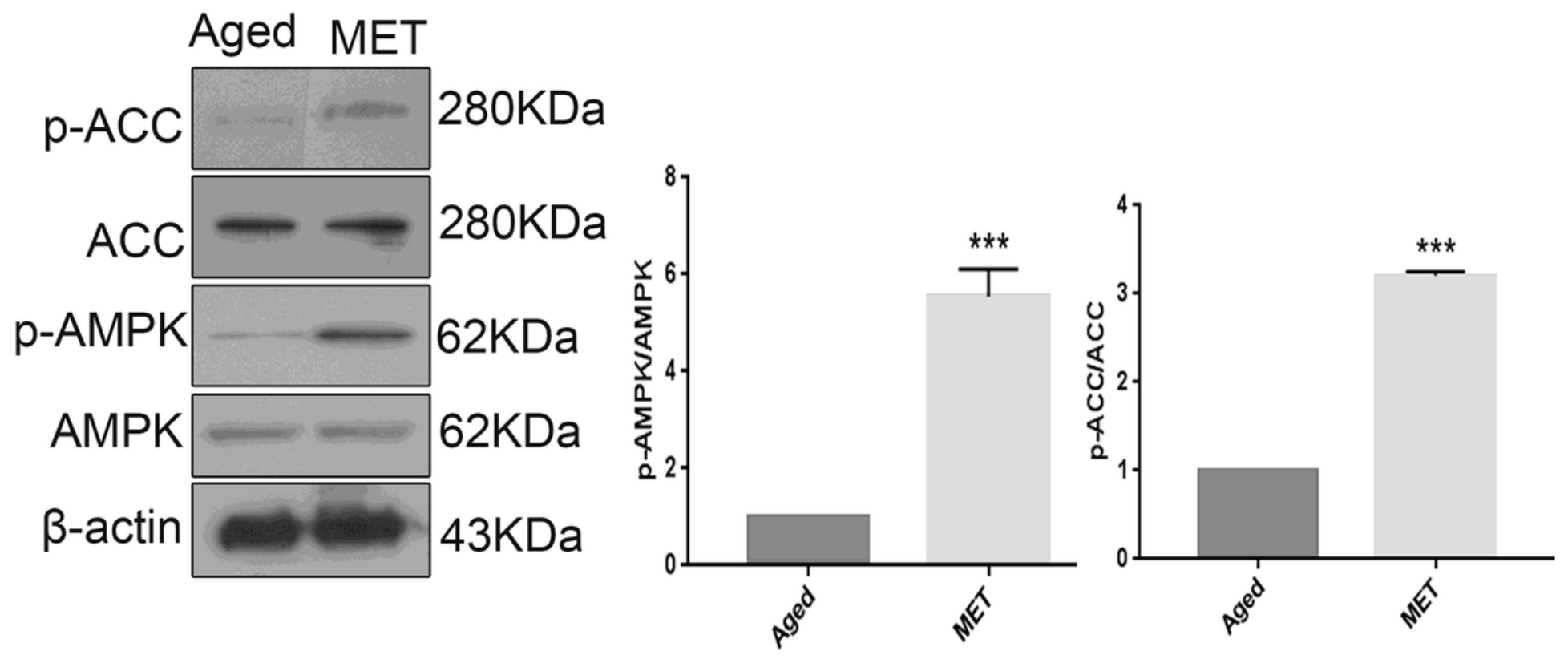

Figure 4

Chronic low-dose MET administration attenuated the senescence of lens epithelial cells in naturally aged mice via AMPK activation. (a). Representative images from IHC assays against p-AMPK in the Aged (left) and the MET (right). (b). Representative images from IHC assays against p-ACC in the Aged (left) and the MET (right). (c). Western blot analysis of AMPK, p-AMPK, ACC, p-ACC and $\beta$-actin in the Aged (left) and the MET (right). Data were shown as mean \pm SD and are representative of 3 independent experiments. $\star \star \star, ~ p<0.001$ compared to the Aged. The bar represents $20 \otimes \mathrm{m}$. 
a

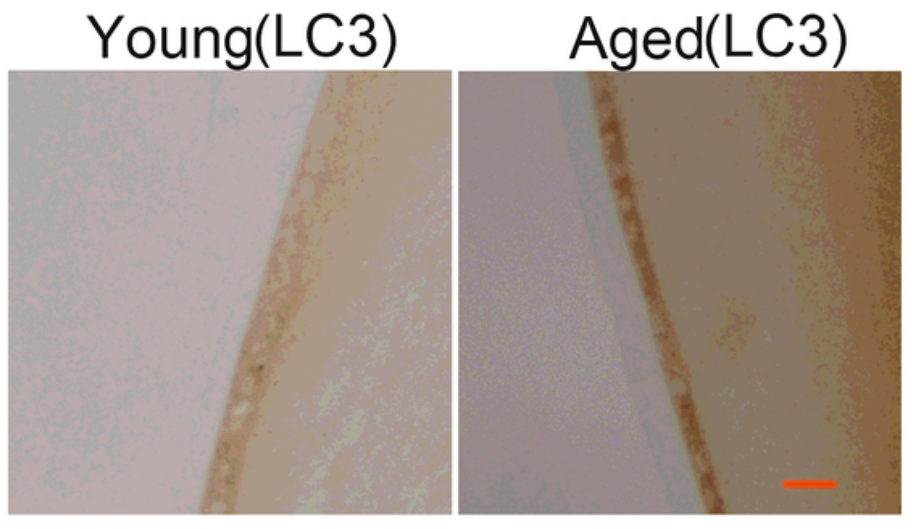

b

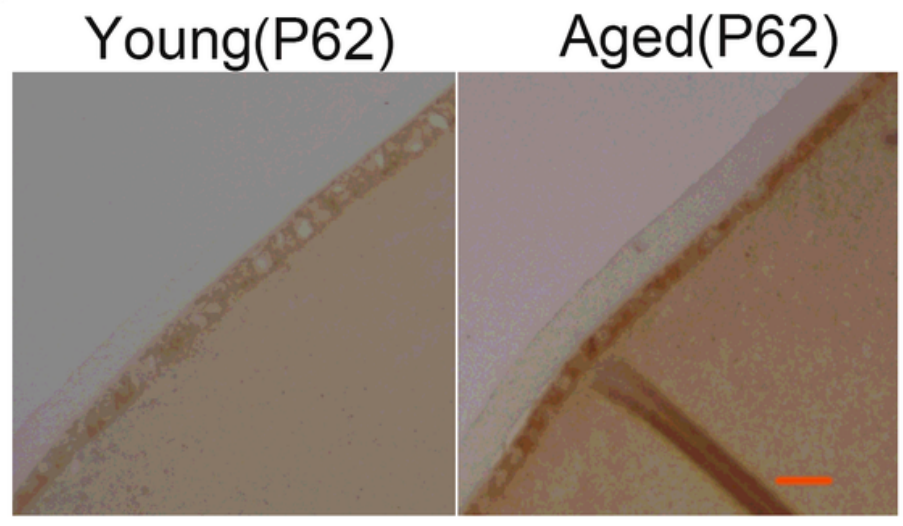

C

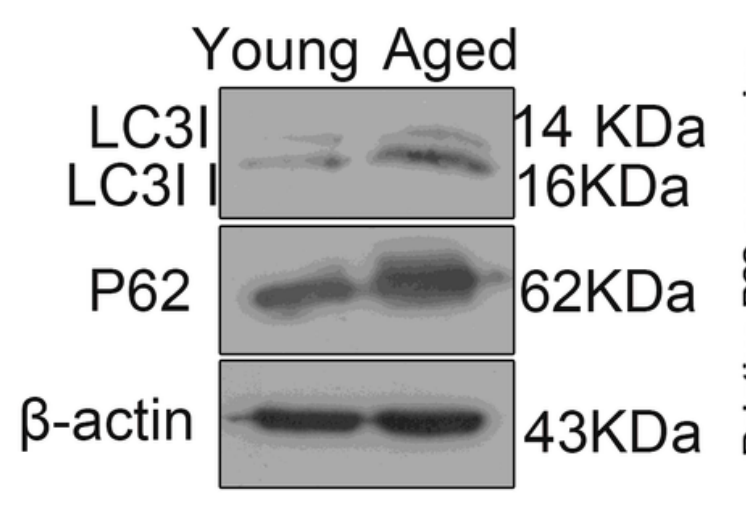

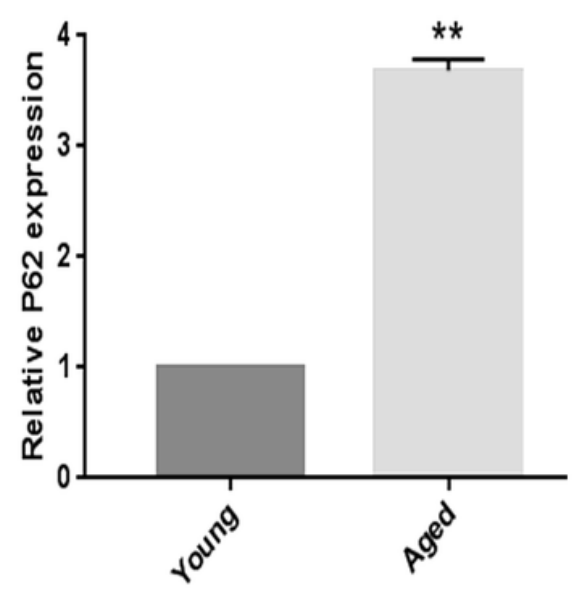

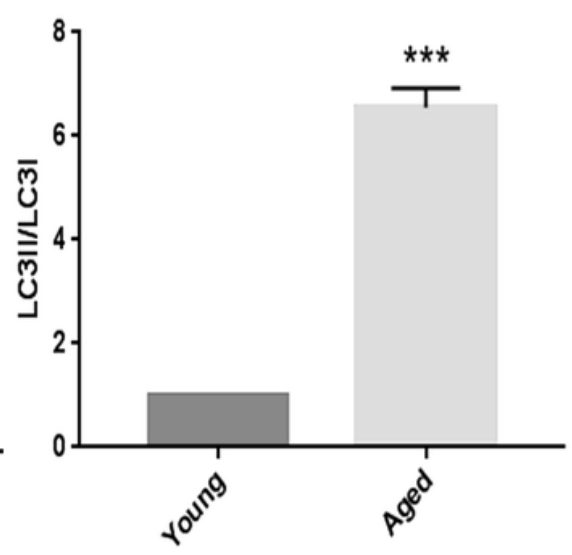

Figure 5

Autophagic flux was impaired in lens epithelial cells of naturally aged mice. (a). Representative images from IHC assays against LC3 in the Young (left) and the Aged (right). (b). Representative images from IHC assays against P62 in the Young (left) and the Aged (right). (c). Western blot analysis of LC3, P62 and $\beta$ actin in the Young and the Aged. Data were shown as mean \pm SD and are representative of 3 independent experiments. ${ }^{\star * \star}, \mathrm{p}<0.001$ compared to the Young. The bar represents $20 \rrbracket \mathrm{m}$. 
a

\section{Aged(LC3)}

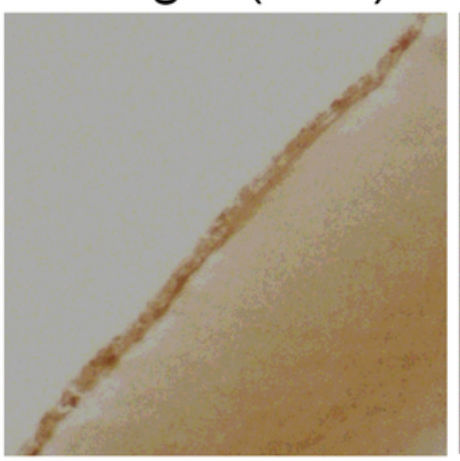

b
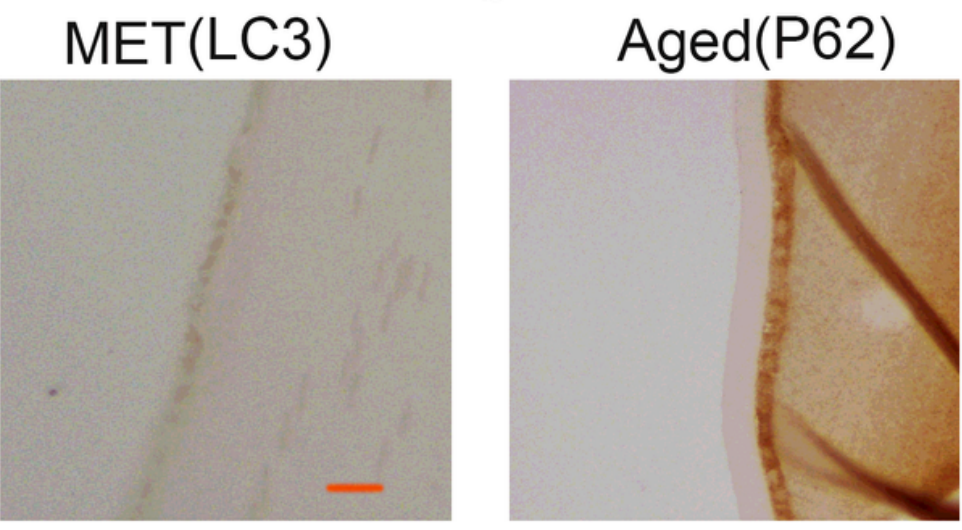

$\operatorname{MET}(\mathrm{P} 62)$

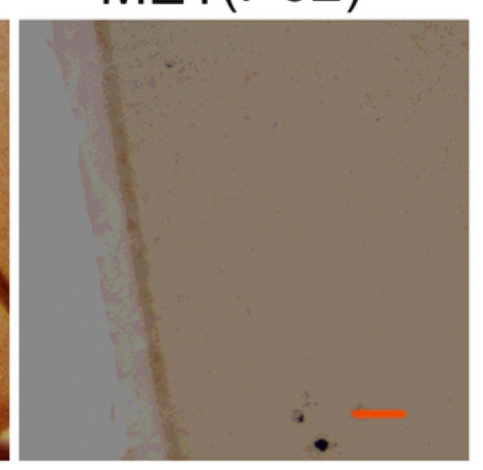

C
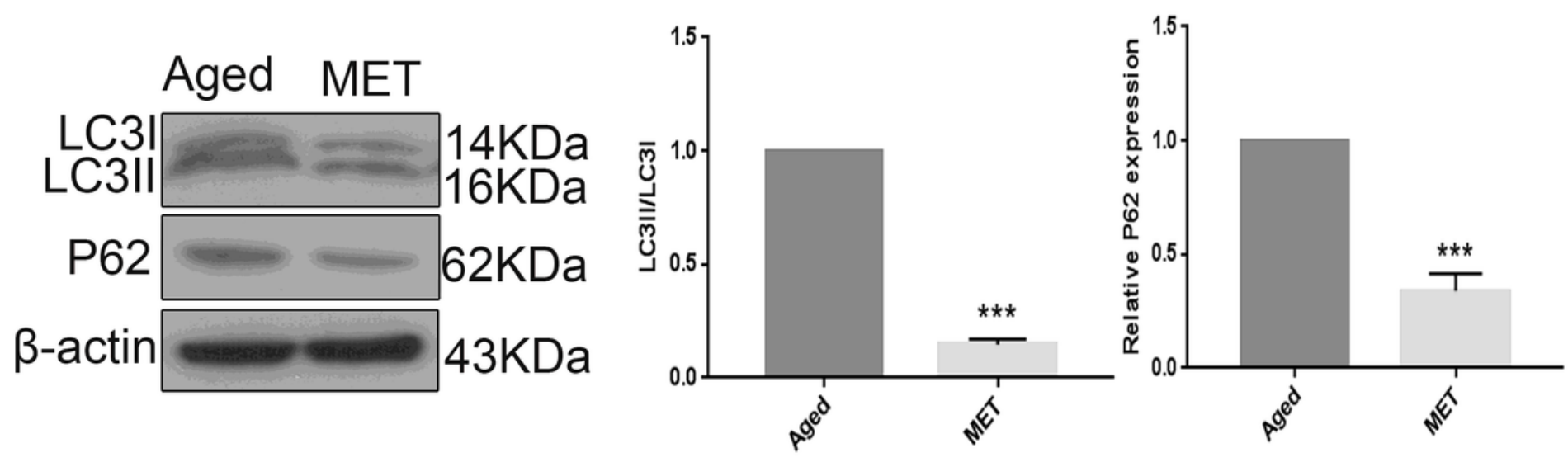

Figure 6

Chronic low-dose MET administration restored the autophagic flux to attenuate the senescence of lens epithelial cells in naturally aged mice. (a). Representative images from IHC assays against LC3 in the Aged (left) and the MET (right). (b). Representative images from IHC assays against P62 in the Aged (left) and the MET (right). (c). Western blot analysis of LC3, P62 and $\beta$-actin in the Aged (left) and the MET (right). Data were shown as mean \pm SD and are representative of 3 independent experiments. ${ }^{* \star}, p<0.001$ compared to the Aged. The bar represents $20 \bigotimes \mathrm{m}$. 